\title{
Efeito da crioterapia de imersão sobre a remoção do lactato sanguíneo após exercício
}

\author{
Effect of immersion cryotherapy on blood lactate clearance \\ after exercise
}

1. Universidade de Caxias do Sul. Laboratório do Movimento Humano. Instituto de Medicina do Esporte. Caxias do Sul, RS. Brasil.

2. Universidade de Caxias do Sul. Curso de Fisioterapia. Caxias do Sul, RS. Brasil.

3.Universidade de Caxias do Sul. Curso de Educação Física. Caxias do Sul, RS. Brasil.

Recebido em 11/08/08 Revisado em 18/12/08 Aprovado em 27/04/09
Resumo - O objetivo deste estudo foi analisar o efeito da crioterapia de imersão sobre a remoção do lactato sanguíneo, um importante parâmetro fisiológico relacionado à fadiga muscular, após exercício de alta intensidade. Para tanto, quinze atletas de futebol (15 a 17 anos) foram divididos em Grupo Imersão (GI, n=7) e Grupo Controle (GC, n=8). Os atletas foram submetidos a um protocolo indutor de fadiga muscular (PIFM) por exercício de alta intensidade em ciclo-ergômetro. Após, os atletas do GI realizaram a crioterapia de imersão, por 10 minutos, com os membros inferiores imersos a $5 \pm 1^{\circ} \mathrm{C}$, enquanto os atletas do GC permaneceram 10 minutos em repouso. Foram coletadas amostras de sangue para análise da concentração de lactato previamente ao PIFM (PRÉ), assim como 3, 15 e 25 minutos após o término do exercício (respectivamente PÓS-3, PÓS-15 e PÓS-25). O PIFM elevou as concentrações de lactato sanguíneo dos atletas significativamente e de maneira similar nos dois grupos. No período de recuperação, o $\mathrm{Gl}$ apresentou redução da concentração de lactato de $13,6 \%$ no PÓS-15 e 15,3\%, no PÓS-25, enquanto o GC apresentou 14,6\% e 28,5\% de redução, no PÓS-15 e PÓS-25, respectivamente. Observou-se que a recuperação passiva apresentou decréscimo significativo da concentração de lactato enquanto o mesmo não foi verificado com a crioterapia. A crioterapia de imersão, nos parâmetros adotados pelo estudo, apresentou-se menos efetiva que repouso para a remoção do lactato sanguíneo após exercício de alta intensidade.

Palavras-chave: Crioterapia; Fadiga muscular; Recuperação muscular.

Abstract - The objective of this study was to analyze the effect of immersion cryotherapy on blood lactate removal, an important physiological parameter related to muscle fatigue, after high-intensity exercise. Fifteen soccer athletes (15 to 17 years) were randomized into an immersion group (IG, $n=7)$ and a control group $(\mathrm{CG}, n=8)$. The athletes were subjected to a muscle fatigue inducer protocol (PIFM) on a cycle ergometer. Next, GI athletes underwent immersion cryotherapy for 10 minutes, with the lower limbs being immersed at $5 \pm 1^{\circ} \mathrm{C}$, whereas $\mathrm{CG}$ athletes rested for 10 minutes. Blood samples were collected for the determination of lactate concentration before the PIFM and 3, 15 and 25 minutes after the end of exercise (post-3, post-15 and post-25, respectively). The PIFM resulted in a significant increase of blood lactate concentration in the athletes, which was similar in two groups. During the recovery period, lactate concentration decreased by $13.6 \%$ at post- 15 and by $15.3 \%$ at post- 25 in $\mathrm{IG}$, whereas GC presented a decrease of $14.6 \%$ and $28.5 \%$ decrease at post- 15 and post-25, respectively. Passive recovery resulted in a significant decrease of lactate concentration, whereas the same was not observed for cryotherapy. These results suggest that, for the parameters used in this study, immersion cryotherapy was less effective than rest in the removal of blood lactate after high-intensity exercise.

Key words: Cryotherapy; Muscle Fatigue; Muscle Recovery. 


\section{INTRODUÇÃO}

A fadiga muscular é um fenômeno frequente na rotina de treinamentos e competições de alguns atletas, podendo prejudicar o desempenho e predispor os mesmos a uma série de lesões musculoesqueléticas. Esse prejuízo pode ser transitório, durando minutos ou horas após o exercício, ou ter duração de longos períodos, como vários dias' ${ }^{1}$. Os prejuízos de curta duração resultam de distúrbios metabólicos ocorridos após o exercício de alta intensidade 2 . Já os prejuízos de longa duração podem estar relacionados à lesão tecidual causada pelo exercício e ao fenômeno conhecido como dor muscular tardia ${ }^{3}$.

Várias estratégias terapêuticas, difundidas no meio desportivo, com a finalidade de acelerar o processo de recuperação muscular pós-exercício vêm sendo estudadas como, por exemplo, recuperação ativa $^{4-8}$, crioterapia $a^{3,6,9-12}$, massagem ${ }^{6}$, terapia de contraste térmico ${ }^{13}$, hidroterapia ${ }^{14}$, alongamento ${ }^{1}$, terapia de oxigênio hiperbárico ${ }^{15}$, anti-inflamatórios não-esteroidais ${ }^{16} \mathrm{e}$ eletroestimulação ${ }^{17}$.

Embora alguns autores ${ }^{1,2,18,19}$ questionem a validade da concentração de lactato como parâmetro para determinar a recuperação muscular pós-exercício, este método tem sido amplamente utilizado com esta finalidade ${ }^{4-8}$. Sabe-se, por exemplo, que a recuperação ativa acelera a velocidade de remoção do lactato do músculo e da circulação sanguínea ${ }^{4,6,7,20}$, havendo estudos ${ }^{5,8}$ que ainda sugerem que tal modalidade terapêutica possa incrementar o desempenho subsequente.

A crioterapia, definida como a utilização de aplicações de gelo ou frio com propósitos terapêuticos ${ }^{21}$, é outra técnica de recuperação amplamente difundida, especialmente, entre atletas de elite ${ }^{1}$. No meio acadêmico, estudos envolvendo crioterapia de imersão já utilizaram desde 10 até 193 minutos, com temperaturas variando de $1^{\circ} \mathrm{Ca} 15^{\circ} \mathrm{C}^{22-24}$. $\mathrm{Na}$ prática desportiva, a crioterapia de imersão consiste na colocação de determinada quantidade de gelo em um balde associado à água (normalmente com temperatura inferior a $15^{\circ} \mathrm{C}$ ), onde os atletas mergulham seus segmentos corporais por quantidades de tempo variáveis ${ }^{25}$.

Embora largamente utilizada na prática desportiva, a efetividade da crioterapia para fins de recuperação muscular pós-exercício ainda carece de evidências científicas ${ }^{1}$. Após extensa busca nas principais bases de dados, não foram encontrados estudos que avaliem o efeito da crioterapia sobre a cinética do lactato sanguíneo. Assim, o presente estudo objetivou analisar o efeito da crioterapia de imersão sobre a remoção de lactato sanguíneo após exercício de alta intensidade.

\section{PROCEDIMENTOS METODOLÓGICOS}

\section{Aspectos Éticos}

O estudo foi aprovado pelo Comitê de Ética em Pesquisa da Universidade do Vale do Paraíba (H229/ $\mathrm{CEP} / 2007)$, estando de acordo com os princípios éticos previstos na resolução número 196/96 do Conselho Nacional de Saúde.

\section{Amostra}

A amostra foi constituída por atletas do sexo masculino, da equipe de futebol da Universidade de Caxias do Sul, com idades entre 15 e 17 anos, federados e participantes de campeonatos de nível estadual e nacional. A rotina do clube incluía cinco sessões de treino semanal, com uma média de duas horas por sessão, além de jogos realizados aos finais de semana.

Como critérios de inclusão, foram adotados: fazer parte da equipe realizando treinamento de forma sistematizada por um período mínimo de um ano; atuar nas chamadas posições de linha, ou seja, foram excluídos os goleiros por apresentarem capacidade aeróbia diferenciada em função das demandas exigidas pela posição em campo; estar participando ativamente das atividades de treinamento e jogos da equipe; não apresentar lesões musculoesqueléticas ou qualquer condição especial de saúde (febre, virose, utilização de fármacos anti-inflamatórios); não apresentar lesões musculoesqueléticas em membros inferiores, responsáveis por afastamento dos treinamentos com data inferior a 30 dias prévios à realização do estudo; apresentar o Termo de Consentimento Livre e Esclarecido assinado pelo responsável previamente à realização dos testes.

Um total de 15 atletas preencheu todos os critérios para participar do estudo, apresentando média de idade de $16,0 \pm 0,8$ anos, $66,8 \pm 6,2 \mathrm{~kg}$ de massa corporal e $174,0 \pm 4,0 \mathrm{~cm}$ de estatura. Os participantes foram aleatoriamente divididos em dois grupos. Após a aleatorização, foi verificada a similaridade entre os grupos, através de um teste $\mathrm{t}$-Student para amostras independentes, para as características supracitadas (idade, massa corporal e estatura) com a finalidade de evitar interferência destas variáveis nos resultados. Não foram encontradas diferenças estatisticamente significantes entre os grupos para os referidos parâmetros, obtendo assim: Grupo Controle (GC), oito atletas (dois 
laterais, dois zagueiros, dois meio-campistas e dois atacantes), 16,0 $\pm 0,8$ anos, $66,1 \pm 5,4 \mathrm{~kg}, 173,0 \pm 5,0$ cm; e Grupo Imersão (GI), sete atletas (dois laterais, um zagueiro, dois meio-campistas e dois atacantes), $16,0 \pm 0,8$ anos, $67,5 \pm 7,4 \mathrm{~kg}, 174,0 \pm 4,0 \mathrm{~cm}$.

\section{Coleta de dados}

O experimento desenvolveu-se no Laboratório do Movimento Humano da Universidade de Caxias do Sul (LMH-UCS), em ambiente climatizado a $24^{\circ} \mathrm{C}$. Após a chegada dos atletas ao laboratório, os mesmos permaneceram sentados por um período mínimo de 10 minutos e, então, foi realizada a primeira coleta sanguínea, estimando o valor de repouso do lactato sanguíneo dos atletas pré-exercício (PRÉ). Foi utilizado o lancetador Accu-Chek Soft Clix ${ }^{\circledR}$ II (Roche, Alemanha) e as lancetas descartáveis da mesma marca e procedência para coleta do sangue da polpa digital dos participantes, sendo a concentração de lactato sanguínea determinada pelo analisador de lactato portátil Accusport ${ }^{\circledR}$ Lactate (Roche, Alemanha).

Na sequência, os voluntários foram submetidos a uma sessão padronizada de preparação para o exercício. Inicialmente, foi realizado o alongamento ativo, por 30 segundos, dos músculos flexores e extensores de joelho, adutores e abdutores de quadril, e flexores plantares, totalizando 4 a 5 minutos de alongamento. Em seguida, os atletas foram encaminhados para o ciclo-ergômetro no qual realizaram um procedimento de aquecimento muscular, durante cinco minutos, de exercício sem carga a aproximadamente $80 \mathrm{rpm}$.

Após o término do aquecimento, foi realizado o protocolo indutor de fadiga muscular (PIFM). Para tal, utilizou-se o ciclo-ergômetro Biotec 2100 AC da marca CEFISE, com o software Ergometric 6.0, para a compilação e análise dos dados. O PIFM adotado, proposto por Martin et al. ${ }^{6}$, foi constituído por três sessões do Teste de Wingate que consiste em 30 segundos de exercício supramáximo em cicloergômetro, com uma carga correspondente a 7,5\% da massa corporal do indivíduo, sendo validado internacionalmente como um método para avaliar a capacidade anaeróbia. Foram consideradas, para posterior análise, a Potência Pico e Potência Média (em valores absolutos e relativos à massa corporal), além do Índice de Fadiga (em valores percentuais). Para as duas primeiras variáveis, foi utilizado o maior valor obtido nos três testes realizados. Já em relação ao Índice de Fadiga, foi utilizado o valor médio das três sessões do Teste de Wingate, calculado pelo próprio software.
Após o PIFM, os atletas permaneceram sentados no ciclo-ergômetro, em repouso, sendo que uma nova amostra de sangue foi coletada três minutos após o término do exercício (PÓS-3). Em seguida, os participantes foram encaminhados para a modalidade de recuperação (crioterapia ou repouso-controle), que fora iniciada cinco minutos após o término do PIFM.

Os atletas do Grupo Controle (GC) permaneceram em repouso, deitados em uma maca, em posição supina, por 10 minutos. Os atletas do Grupo Imersão (GI) permaneceram em ortostase, dentro de um tonel de metal $(100 \mathrm{~cm}$ de altura, $60 \mathrm{~cm}$ de diâmetro e capacidade para 250 litros) com água e gelo, de modo que seus membros inferiores permaneceram imersos (estando o nível de imersão imediatamente abaixo das gônadas), por 10 minutos, sob uma temperatura de $5 \pm 1^{\circ} \mathrm{C}$. A temperatura da água foi verificada de maneira constante por meio de termômetro sub-aquático e ajustada, quando necessário, pela adição de gelo industrializado em cubos ou água morna.

Em consequência da falta de padronização quanto aos parâmetros a serem utilizados na crioterapia de imersão, optou-se pela adoção do tempo de 10 minutos, baseado em Andrews et al. ${ }^{26}$, que propõem tempos entre 5 e 15 minutos para a crioterapia. Para a definição da temperatura, utilizou-se como base o estudo de Sellwood et al. ${ }^{12}$, que definiram como ice water immersion a mistura de água e gelo a uma temperatura de, aproximadamente, $5^{\circ} \mathrm{C}$. $\mathrm{O}$ intervalo de 5 minutos entre o fim do PIFM e o início da crioterapia de imersão foi escolhido por se considerar um tempo passível de ser aplicado na prática desportiva.

Finalizada a modalidade de recuperação, ocorreu uma nova coleta de sangue, 15 minutos após o término do PIFM (PÓS-15). O atleta permaneceu sentado por mais 10 minutos para a coleta da última amostra sanguínea, realizada 25 minutos após o término do PIFM (PÓS-25), finalizando o procedimento.

\section{Análise Estatística}

Inicialmente, todas as variáveis foram testadas quanto à normalidade da distribuição pelo teste de Shapiro-Wilk. Para as variáveis de caracterização da amostra (idade, massa corporal e estatura) e do desempenho dos atletas no PIFM (Potência Máxima, Potência Média e Índice de Fadiga), foi utilizado um teste $t$-Student para amostras independentes na comparação intergrupos.

Para a comparação intergrupos das concentrações de lactato sanguíneo, durante os quatro 
momentos analisados (PRÉ, PÓS-3, PÓS-15 e PÓS25), utilizou-se a ANOVA Simples. Para observar o comportamento de cada grupo, isoladamente, durante o procedimento, utilizou-se a ANOVA para medidas repetidas. Em ambas as análises utilizou-se o post-hoc de Bonferroni.

Optou-se pela realização de uma comparação das modalidades por meio de uma análise normalizada dos dados, considerando o valor PÓS-3 como $100 \%$. Assim, novamente foi utilizada a ANOVA Simples para a comparação intergrupos e a ANO. VA para medidas repetidas, na comparação intragrupo, ambas seguidas do post-hoc de Bonferroni.

Atesta-se que para todas as variáveis analisadas foi adotado o nível de significância de 5\% $(\mathrm{p}<0,05)$.

\section{RESULTADOS}

Os resultados estão expostos em forma de valores médios e respectivos desvios-padrão. A Tabela 1 apresenta os valores apresentados pelos atletas (GI, GC e a média geral) para as variáveis de desempenho no PIFM. Na comparação intergrupos, não foi encontrada diferença estatisticamente significante em nenhuma das cinco variáveis analisadas.

Tabela 1. Desempenho dos atletas no protocolo indutor de fadiga muscular.

\begin{tabular}{lccc}
\hline & Gl & GC & Média \\
\hline PPA (W) & $747,5 \pm 102,4$ & $726,8 \pm 98,7$ & $736,4 \pm 97,3$ \\
\hline PPR (W/kg) & $11,1 \pm 0,7$ & $11,0 \pm 1,1$ & $11,0 \pm 0,9$ \\
\hline PMA (W) & $469,8 \pm 54,8$ & $448,4 \pm 34,6$ & $458,4 \pm 44,8$ \\
PMR (W/kg) & $7,0 \pm 0,4$ & $6,8 \pm 0,3$ & $6,9 \pm 0,4$ \\
IF (\%) & $65,8 \pm 13,0$ & $65,6 \pm 10,6$ & $65,7 \pm 11,3$ \\
\hline
\end{tabular}

PPA - Potência Pico Absoluta; PPR - Potência Pico Relativa; PMA - Potência Média Absoluta; PMR - Potência Média Relativa; IF - Índice de Fadiga.

Observou-se, na coleta de sangue prévia ao teste (PRÉ), concentrações médias de lactato san-

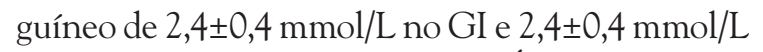
no GC. Na coleta após o PIFM (PÓS-3), o GI apresentou valor médio de $14,2 \pm 4,0 \mathrm{mmol} / \mathrm{L}$, enquanto

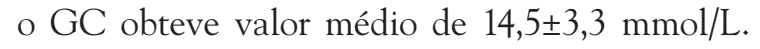
Não foram observadas diferenças estatisticamente significantes entre os grupos em nenhum dos dois momentos; entretanto, em ambos foi verificado um aumento significativo $(\mathrm{p}<0,05)$ da concentração de lactato em função do PIFM. Na fase de recuperação (PÓS-15 e PÓS-25), o GI apresentou os respectivos valores médios de $11,5 \pm 2,3$ e $11,5 \pm 1,9 \mathrm{mmol} / \mathrm{L}$, enquanto o GC obteve valores médios de 12,1 $\pm 2,5$ e 10,0 $\pm 2,5 \mathrm{mmol} / \mathrm{L}$. Não houve diferenças na comparação intergrupos em nenhum dos momentos. Entretanto, na análise intragrupo, o GC apresentou remoção significativa $(\mathrm{p}<0,05)$ do lactato sanguíneo no PÓS-25 em relação ao PÓS-3 (Figura 1).
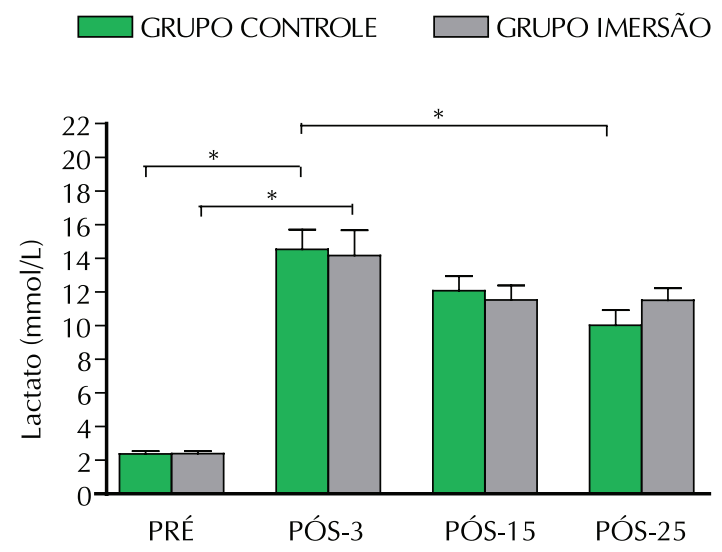

Figura 1. Comportamento das concentrações médias de lactato sanguíneo nos quatro momentos de análise do estudo. $\left({ }^{*} p<0,05\right)$

Com o intuito de realizar uma comparação igualitária e utilizando medidas padronizadas entre a efetividade de cada técnica sobre a remoção do lactato sanguíneo, optou-se por também avaliar os resultados em valores percentuais. Para tal, considerou-se a concentração de lactato imediata, após o PIFM (PÓS-3), como valor correspondente a $100 \%$, conforme proposto por Martin et al. ${ }^{6}$. Dessa maneira, pode-se observar em valores percentuais o efeito exato da crioterapia de imersão e do repouso sobre a remoção do lactato sanguíneo.

A Figura 2 expressa os valores médios percentuais relativos à concentração de lactato sanguíneo nos três momentos posteriores ao PIFM.
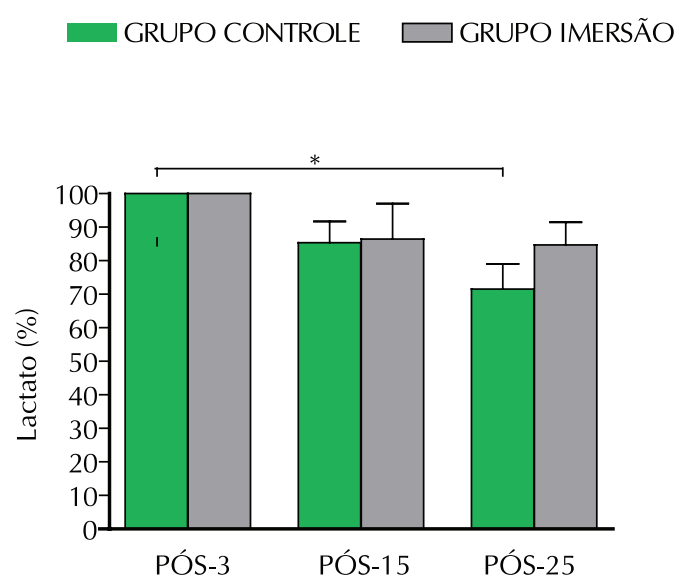

Figura 2. Concentrações médias de lactato sanguíneo em valores percentuais durante o período de recuperação. $\left({ }^{*} p<0,05\right)$ 
Considerando ambos os grupos com valores de 100\%, em PÓS-3, observa-se, no GI, uma redução para $86,4 \pm 28,0 \%$, em PÓS-15 e para $84,7 \pm 17,9 \%$, em PÓS-25, enquanto no GC houve queda para $85,3 \pm 18,0 \%$ em PÓS-15 e 71,5 $21,3 \%$ em PÓS- 25 . Novamente, na comparação intergrupos, não foi observada diferença estatisticamente significante em nenhum dos momentos analisados. Porém, na análise intragrupo, a remoção de lactato sanguíneo mostrou-se significativa $(p<0,05)$ no GC.

\section{DISCUSSÃO}

Alguns aspectos denotam a homogeneidade da amostra utilizada e elevam a confiabilidade dos resultados obtidos: i) todos os participantes do estudo possuem uma mesma rotina semi-profissional de treinamento; ii) os dois grupos não apresentam diferenças significativas nas variáveis: idade, massa corporal e estatura; iii) as concentrações de lactato sanguíneo em repouso (PRÉ) revelam elevada similaridade entre os grupos; iv) o desempenho de ambos os grupos perante o PIFM adotado foi semelhante, não sendo encontradas diferenças estatísticas para nenhuma das mensurações fornecidas pelo software utilizado; v) os resultados das coletas de lactato imediato após o exercício (PÓS-3) também revelam a inexistência de diferença significativa entre os grupos, o que comprova que o protocolo proporcionou respostas similares na cinética do lactato entre os atletas.

Salienta-se que o PIFM adotado mostrou-se efetivo, fato justificado pelo incremento da concentração de lactato sanguíneo em, aproximadamente, seis vezes do valor PRÉ para o valor PÓS-3. Este significante incremento está relacionado à participação do metabolismo anaeróbio lático no exercício realizado. Segundo os cálculos de Serresse et al...27, durante o Teste de Wingate ocorrem contribuições de $23 \%$ do metabolismo aeróbio, $49 \%$ do metabolismo glicolítico (anaeróbio lático) e 28\% do sistema ATP-CP (anaeróbio alático). No entanto, a contribuição de cada um dos sistemas energéticos parece ser diferente conforme o gênero (masculino ou feminino $)^{28}$ e o tipo de treinamento a que é submetido $^{29}$. Apesar de poucos trabalhos analisarem protocolos de sprints intervalados, como o utilizado no presente estudo, aparentemente, a participação do metabolismo aeróbio é incrementada a cada nova repetição ${ }^{30}$.

Embora alguns autores ${ }^{1,2,18,19}$ questionem a validade da concentração de lactato como parâmetro para determinar a recuperação muscular pós-exercí- cio, este método tem sido amplamente utilizado com esta finalidade ${ }^{4-8}$. Robergs et al. ${ }^{18}$, em uma extensa revisão sobre acidose metabólica, enfatizam que a relação entre o incremento de ácido lático e o decréscimo do $\mathrm{pH}$ não devem ser interpretados como uma relação de causa e efeito. Porém, admitem que o acúmulo de lactato seja um bom indicador indireto do incremento do próton $\mathrm{H}^{+}$e a diminuição do $\mathrm{pH}$ celular e sanguíneo, fatores promotores da acidose metabólica e subsequente fadiga muscular. Ou seja, os mecanismos exatos relacionados aos fenômenos da fadiga muscular e da acidose metabólica, assim como a participação do ácido lático neste processo, não estão completamente elucidados. Entretanto, sugere-se que, ao passo que uma técnica de recuperação aumenta a remoção deste subproduto da glicólise, aumenta também a remoção de outras substâncias decorrentes do processo. Logo, quanto maior a taxa de remoção destas substâncias pela circulação sanguínea, mais rapidamente o músculo retorna ao seu estado basal, o que teoricamente o torna mais apto para realizar uma nova atividade com máximo desempenho.

Os resultados obtidos no presente estudo sugerem que a crioterapia de imersão não acelerou a velocidade de remoção do lactato sanguíneo dos atletas avaliados, mostrando-se, inclusive, menos efetiva que o repouso. Em estudo metodologicamente semelhante, Martin et al. ${ }^{6}$ compararam o efeito da massagem, da recuparação ativa e do repouso sobre a remoção do lactato após PIFM idêntico ao do presente estudo. Entretanto, a natureza da amostra utilizada limita a comparação com os nossos resultados. Enquanto os ciclistas adultos de nível competitivo, do estudo de Martin et al. ${ }^{6}$, apresentaram uma redução de $40 \%$ da concentração de lactato com o repouso de 25 minutos após o PIFM, os futebolistas adolescentes do nosso grupo controle apresentaram menos de 30\% de remoção no mesmo período. Acredita-se que tal diferença esteja relacionada ao fato de atletas com maior nível de aptidão física apresentarem maior velocidade de remoção do lactato ${ }^{31}$. Também deve ser mencionada uma provável influência da especificidade, já que os ciclistas certamente apresentam melhor adaptação ao exercício em ciclo-ergômetro, o que incrementa sua capacidade de recuperação no protocolo adotado.

Selwood et al. ${ }^{12}$ não encontraram diferença entre o efeito da crioterapia e do repouso, após um protocolo de exercícios excêntricos para membros inferiores, sobre a creatina quinase, uma enzima marcadora de lesão tecidual, assim como em re- 
lação à dor muscular tardia, edema de membro inferior, testes isométricos e de desempenho. Da mesma forma, trabalhos com indivíduos do sexo masculino treinados ${ }^{10}$, destreinados ${ }^{11}$ e indivíduos do sexo feminino ${ }^{9}$ não têm encontrado incrementos de desempenho subsequente à crioterapia realizada após exercícios de alta intensidade.

Assim, embora não sejam encontradas evidências de que a crioterapia de imersão altere parâmetros fisiológicos (como a concentração de lactato ou da enzima creatina quinase $\mathrm{e}^{12}$ ) ou aumente o desempenho subsequente ${ }^{9-11}$, as sensações de bem estar e relaxamento muscular relatadas por uma série de atletas podem estar relacionadas a outros efeitos comprovados da crioterapia.

O efeito inicial da crioterapia se constitui na redução da temperatura ${ }^{3,21}$. Com a imersão de grandes áreas corporais, esta diminuição é observada não só em nível local, mas também da temperatura corporal sistêmica ${ }^{32}$. Também devido à aplicação de baixas temperaturas, ocorre a diminuição do metabolismo por uma reação do organismo em diminuir a demanda energética e de oxigênio do local. Assim, a diminuição do fluxo sanguíneo é imediata ao início da crioterapia ${ }^{21}$.

Apesar de muita polêmica sobre o assunto, estudos $^{24,25}$ já comprovaram que a vasodilatação que ocorre após algum tempo de aplicação do gelo, identificada primeiramente por Lewys ${ }^{33}$, não supera o estado de repouso anterior ao início da crioterapia. Ou seja, o incremento do fluxo de sangue após alguns minutos de crioterapia existe, porém não representa um aumento da circulação sanguínea local quando comparado ao fluxo normal. Assim, como enfatizado por Knight ${ }^{21}$, o efeito do gelo sobre a circulação é a vasoconstrição. E provavelmente seja este efeito vasoconstritor que melhor explique a ineficiência da crioterapia de imersão em incrementar a velocidade de remoção do lactato sanguíneo.

Uma provável justificativa para a sensação de relaxamento decorrente da crioterapia de imersão seja o comprovado efeito analgésico desta modalidade $^{3,21}$. Embora não se tenha um consenso quanto aos mecanismos exatos pelos quais o frio age na redução da dor, Knight et al. ${ }^{21}$ citam uma série de estudos que sugerem efeitos do frio que podem explicar tal fenômeno: redução da transmissão nervosa nas fibras de dor; redução da excitabilidade nas terminações nervosas livres; redução no metabolismo tecidual; promoção de transmissão assincrônica nas fibras de dor; elevação do limiar de dor; liberação de endorfinas; e inibição de neurônios espinhais. Além disso, outros efeitos da crioterapia que podem estar relacionados à sensação de relaxamento muscular são as reduções do espasmo muscular ${ }^{21}$ e do edema ${ }^{3}$.

Dentre as limitações do presente estudo, pode-se destacar a inviabilidade de realizar um ensaio clínico cruzado (onde o mesmo indivíduo seria submetido aos dois métodos de recuperação) devido à rotina de treinamentos dos atletas. Tal fato reduziu a expressividade da amostra, já que se fez necessária a criação de dois grupos. Também ressalta-se que a especificidade do gesto em ciclo-ergômetro, não comum à prática do futebol, pode ter influência sobre os resultados. Por fim, sugere-se que futuros estudos correlacionem uma maior quantidade de variáveis (lactato sanguíneo, enzimas marcadoras de lesão, sensação subjetiva do atleta, desempenho subsequente, dor muscular tardia, etc.); e observem efeitos a médio e longo prazo da crioterapia de imersão.

\section{CONCLUSÃO}

Os resultados nos permitem concluir que a crioterapia de imersão, nos parâmetros adotados, mostrou-se menos eficiente que a recuperação passiva (repouso) para a remoção do lactato sanguíneo após exercício de alta intensidade. Assim, a utilização desta modalidade para o objetivo proposto parece ser uma prática equivocada por parte dos profissionais da área. Entretanto, visto alguns efeitos benéficos da crioterapia apresentados na literatura, instiga-se que mais estudos sejam realizados com tal modalidade, visando elucidar outros aspectos relacionados à recuperação muscular após o exercício intenso.

\section{REFERÊNCIAS BIBLIOGRÁFICAS}

1. Barnett A. Using recovery modalities between training sessions in elite athletes. Does it help? Sports Med 2006;36(9):781-796.

2. Westerblad H, Allen DG, Lannergren J. Muscle fatigue: lactic acid or inorganic phosphate the major cause? News Physiol Sci 2002;17(1):17-21.

3. Cheung K, Hume PA, Maxwell L. Delayed onset muscle soreness: treatment strategies and performance factors. Sports Med 2003;33(2):145-164.

4. Taoutaou Z, Granier P, Mercier B, Mercier J, Ahmaidi $\mathrm{S}$, Prefaut C. Lactate kinetics during passive and partially active recovery in endurance and sprint athletes. Eur J Appl Physiol Occup Physiol 1996;73(5):465-470.

5. Ahmaidi SP, Granier Z, Taoutaou J, Mercier H, Dubouchaud, Prefaut C. Effects of active recovery on plasma lactate anaerobic power following repeated intensive 
exercise. Med Sci Sports Exerc 1996;28(4):450-456.

6. Martin NA, Zoeller RF, Robertson RJ, Lephart SM. The comparative effects of sports massage, active recovery, and rest in promoting blood lactate clearance after supramaximal leg exercise. J Athl Training 1998;33(1):30-35.

7. Dotan R, Falk B, Raz A. Intensity effect of active recovery from glycolytic exercise on decreasing blood lactate concentration in prepubertal children. Med Sci Sports Exerc 2000;32(3):564-570.

8. Spierer DK, Goldsmith R, Baran DA, Hryniewicz K, Katz SD. Effects of active vs. passive recovery on work performed during serial supramaximal exercise tests. Int J Sports Med 2004;25(2):109-114.

9. Eston R, Peters D. Effects of cold water immersion on the symptoms of exercise-induced muscle damage. J Sports Sci 1999;17(3):231-238.

10. Howatson G, Van Someren KA. Ice massage: effects on exercise-induced muscle damage. J Sports Med Phys Fitness 2003;43(4):500-505.

11. Howatson G, Gaze D, Van Someren KA. The efficacy of ice massage in the treatment of exercise-induced muscle damage. Scand J Med Sci Sports 2005;15(6):416-422.

12. Sellwood KL, Brukner P, Williams D, Nicol A, Hinman R. Ice-water immersion and delayed-onset muscle soreness: a randomized controlled trial. Br J Sports Med 2007;41(6):392-397.

13. Gill ND, Beaven CM, Cook C. Effectiveness of postmatch recovery strategies in rugby players. Br J Sports Med 2006;40(3):260-263.

14. Dowzer CN, Reilly T, Cable NT. Effects of deep and shallow water running on spinal shrinkage. Br J Sports Med 1998;32(1):44-48.

15. Mekjavic IB, Exner JA, Tesch PA, Eiken O. Hyperbaric oxygen therapy does not affect recovery from delayed onset muscle soreness. Med Sci Sports Exerc 2000;32(3):558-563.

16. Baldwin Lanier A. Use of nonsteroidal anti-inflammatory drugs following exercise-induced muscle injury. Sports Med 2003;33(3):177-185.

17. Lattier G, Millet GY, Martin A, Martin V. Fatigue and recovery after high-intensity exercise. Part II: recovery interventions. Int J Sports Med 2004;25(7):509-515.

18. Robergs RA, Ghiasvand F, Parker D. Biochemistry of exercise-induced metabolic acidosis. Am J Physiol Regul Integr Comp Physiol 2004;287(3):502-516.

19. Cairns SP. Lactic acid and exercise performance: culprit or friend? Sports Med 2006;36(4):279-291.

20. Reilly T, Ekblom B. The use of recovery methods postexercise. J Sports Sci 2005;23(6):619-627.

21. Knight KL. Cryotherapy in Sport Injury Management. Champaign, IL: Human Kinetics; 1995.
22. Miglietta O. Action of cold on spasticity. Am J Phys Med 1973;52(4):198-205.

23. Abramson DI, Chu LS, Tuck S, Lee SW, Richardson G. Effect of tissue temperatures and blood flow on motor nerve conduction velocity. J Am Med Assoc 1966;198(10):1082-1088.

24. Knight KL, Bryan KS, Halvorsen JM. Circulatory changes in the forearm in $1,5,10$ and $15^{\circ} \mathrm{C}$ water (Abstract). Int J Sports Med 1981;4:281.

25. Wilcock IM, Cronin JB, Hing WA. Physiological response to water immersion. A method for sport recovery? Sports Med 2006;36(9):747-765.

26. Andrews J, Harrelson G, Wilk K. Physical rehabilitation of the injured athlete. $3^{\text {rd }}$ ed. Philadelphia: Saunders, 2004.

27. Serresse O, Lortie G, Bouchard C, Boulay MR. Estimation of the contribution of the various energy systems during maximal work of short duration. Int J Sports Med 1988;9(6):456-460.

28. Hill DW, Smith JC. Gender difference in anaerobic capacity: role of aerobic contribution. Br J Sports Med 1993;27(1):45-48.

29. Granier P, Mercier B, Mercier J, Anselme F, Préfaut C. Aerobic and anaerobic contribution of Wingate test performance in sprint and middle-distance runners. Eur J Appl Physiol 1995;70(1):58-65.

30. Spencer M, Bishop D, Dawson B, Goodman C. Physiological and metabolic responses of repeated-sprint activities. Specific to field-based team sports. Sports Med 2005;35(12):1025-1044.

31. Koutedakis Y, Sharp NC. Lactic acid removal and heart rate frequencies during recovery after strenuous rowing exercise. Br J Sports Med 1985;19(4):199-202.

32. Clements JM, Casa DJ, Knight JC, McClung JM, Blake AS, Meenen PM, et al. Ice-water immersion and cold-water immersion provide similar cooling rates in runners with exercise-induced hyperthermia. J Athl Train 2002;37(2):146-150.

33. Lewis T. Observations upon the reactions of the vessels of the human skin to cold. Heart 1930;15(2):177-208.
Endereço para correspondência
Bruno Manfredini Baroni
Universidade de Caxias do Sul (UCS)
Instituto de Medicina do Esporte (IME)
Rua Francisco Getúlio Vargas, 1130 -Bloco 70
CEP: 95070-560 - Caxias do Sul, Brasil -
E-mail: baroni09@yahoo.com.br 